\title{
Mercado del sexo: Reflexiones desde la economía al comercio sexual ${ }^{1}$
}

\section{Carlos Alfonso Laverde Rodríguez ${ }^{2}$}

\section{RESUMEN}

El presente documento es una aproximación al mercado del sexo y de quienes lo desarrollan principalmente (Trabajadores del sexo), identificando los aportes teóricos que se han propuesto desde la economía a través de sus diversos paradigmas, desde marcos teóricos como el institucionalismo, la teoría neoclásica y el capital humano. A través de este documento se vislumbran los vacíos en las explicaciones teóricas tradicionales de la economía en la exploración del comercio y trabajo sexual en el marco del mercado laboral en el mundo contemporáneo, abriendo un campo de discusión sobre fenómenos como la discriminación de género en la ciencia económica.

Palabras Clave: mercado del sexo, discriminación de género, mercado laboral, trabajo sexual, comercio sexual.

\section{Sex Market: Reflections from economics to sex}

\section{ABSTRACT}

This paper is an approach to sex market and those who develop primarily (sex

\footnotetext{
${ }^{1}$ Artículo resultado de reflexión a partir de la investigación "Una aproximación al mercado del sexo desde una perspectiva económica" para optar al título de economista por Carlos Alfonso Laverde y Juan Camilo Fajardo.

${ }^{2}$ Sociólogo y Economista Universidad Santo Tomas. Estudiante de la Maestría en Estudios Políticos y Sociales de la Universidad Nacional Autónoma de México (UNAM) correo electrónico: smials1@ gmail.com
} 
workers), identifying the theoretical contributions that have been proposed since the economy through its various paradigms, from theoretical as institutionalism, the neoclassical theory and human capital. Through this document looming gaps in traditional theoretical explanations of the economy in the exploration of trade and sex work in the context of the labor market in the contemporary world, opening a discussion of field phenomena such as gender discrimination in the economics.

Keywords: sex market, gender discrimination, labor market, sex work, sex trade

\section{Mercado do sexo: Reflexões a partir da economia ao sexo}

\section{RESUMO}

Este trabalho é uma abordagem para o mercado do sexo e aqueles que desenvolvem principalmente (profissionais do sexo), identificando as contribuições teóricas que têm sido propostas desde a economia através de seus vários paradigmas, a partir de teóricos como institucionalismo, a teoria neoclássica e capital humano. Através deste documento iminente lacunas em explicações teóricas tradicionais da economia na exploração do comércio e do trabalho sexual no contexto do mercado de trabalho no mundo contemporâneo, abrindo uma discussão sobre os fenômenos de campo, tais como a discriminação de gênero na economia.

Palavras-chave: mercado do sexo, a discriminação de gênero, mercado de trabalho, trabalho sexual, o comércio do sexo.

\section{Introducción}


El mercado del sexo se ha acomodado a las necesidades de las modernas sociedades llegando a un grado alto de sofisticación. Actualmente, por medio de internet se puede elegir un cuerpo femenino o masculino o desde el mismo sitio de residencia se pueden tener encuentros sexuales virtuales, sumado a las diversas formas en las que se pueden acceder a servicios de tipo sexual como table-dance, shows, revistas pornográficas, videos, entre otras tantas variedades mostrando un panorama amplio de categorías del trabajo sexual contemporáneo que, por una parte, ha venido mejorando los ingresos de los empresarios (industria) del sexo y, por otra, ha desencadenado problemáticas en el mundo como la trata de personas y/o la explotación sexual con fines lucrativos utilizando a los menores de edad.

La economía como ciencia social, ha intentado desde sus posturas teóricas dar cuenta del fenómeno del mercado del sexo aunque de una forma tangencial; desde Adam Smith con su obra "Una investigación sobre la naturaleza y causas de la Riqueza de la Naciones" publicada en 1776, se formularon lineamientos sobre las implicaciones de la sexualidad y sus repercusiones en el estudio de los fenómenos económicos, pasando a otros autores de la historia del pensamiento económico como John Stuart Mill (1869), Tullock \& McKenzie (1980) y más recientemente, los planteamientos realizados por Gary Becker $(1977,1993,2008)$ y Douglas North (1971) desde una visión del capital humano y del institucionalismo, respectivamente. En Colombia el fenómeno es tratado, entre otros, por los economistas Mauricio Rubio $(2006,2008)$ y Tirado $(2010,2011)$ quienes han realizado diversos acercamientos desde una perspectiva de la economía del mercado del sexo con la ilegalidad y sus implicaciones.

A partir de lo anterior, el estudio del mercado del sexo desde una perspectiva económica es imperante para comprender el campo del trabajo sexual como una manifestación de la racionalidad de las necesidades sexuales que permite establecer intercambios que, en un primer momento, no se relacionan más que en un plano simbólico pasando al mercado del sexo en el cual se pacta un 
intercambio monetario explicito, desencadenando una serie de consecuencias que afectan tanto a las personas que la ejercen como al contexto en el que se desenvuelven. Es así como se revela la importancia del análisis económico de un fenómeno social que ante su magnitud e impacto relaciona y debe interesar la intervención del Estado como garante primario de la satisfacción de derechos como el trabajo, la dignidad y la salud, que han estado en vilo para la población que ejerce el trabajo sexual por cuenta de la carga moral que implica su ejercicio.

Así mismo la revisión teórica sobre el mercado del sexo requiere perspectivas que aporten tanto a la construcción académica como a los actos legislativos en un momento coyuntural de la realidad nacional en la que el trabajo sexual se ha convertido recientemente en un fenómeno mediático. ${ }^{3}$

\section{Fundamentos y características del mercado del sexo}

El mercado y trabajo del sexo son conceptos que tienen su desarrollo especialmente en las últimas décadas, por lo que si se quiere indagar sobre sus características y fundamentos hay que recurrir al concepto de prostitución, fenómeno que no es reciente en la historia, tomando diferentes formas como respuesta a las disposiciones sociales y ordenándose de acuerdo al orden social establecido como un paralelo al desarrollo moral, jugando en espacios ocultos pero visibles para la sociedad, marcando el camino del mercado del sexo en todas sus manifestaciones.

En el contexto colombiano, la Contraloría de Bogotá (2004) concibe la prostitución cuando "cualquier hombre o mujer que lleva a cabo el coito con compañero

\footnotetext{
${ }^{3}$ En mayo del 2012 llevándose a cabo la Cumbre de las Américas en la ciudad de Cartagena, se desató un escándalo por cuenta de varios agentes de seguridad de Estados Unidos quienes demandaron la prestación de servicios sexuales, generando un escándalo mediático debido a las quejas de la trabajadora sexual quien manifestó que uno de los agentes con el que estuvo no cumplió las condiciones de pago. En: http://www.elespectador.com/impreso/internacional/articulo-342417-los-malos-habitos-del-servicio-secreto-deestados-unidos, obtenida el 01 de abril de 2012.
} 
compañera, en cuya elección se excluye el factor emocional" (p. 7), coincidiendo más o menos en las mismas características de un intercambio sexual a cambio de dinero. Edlund \& Korn (2002) destacan que desde la perspectiva del cliente, la prostitución es la forma de tener sexo no reproductivo mediante una transacción normalmente monetaria, por lo cual no podría afirmarse que es la venta del cuerpo, sino de la prestación de un servicio.

Tirado (2010) ha señalado que la prostitución podría definirse como un ser que se entrega sexualmente a cambio de algo, señalando que en búsqueda de un consenso en una perspectiva internacional la prostitución es un término que "alude a la venta de actos sexuales por dinero o servicios" (Tirado, 2010, p.55).

Frente a la definición de la prostitución han surgido diferentes concepciones de acuerdo a cada contexto y orientación ideológica, destacándose dos posiciones predominantes. Por una parte, se concibe el ejercicio de la prostitución como la expresión de una relación de poder en la que existe un sometimiento por parte de quien se prostituye, empujada por causa de la necesidad; por otra parte, como lo señala Tirado (2010), como fruto de la libre elección de forma de vida y de fuente de ingresos.

Características y factores de desarrollo de la prostitución como fenómeno social

Existen múltiples causas que han determinando el ejercicio del trabajo sexual, desde un marco social que abre las posibilidades de desarrollo del fenómeno hasta características individuales asociadas a la libre decisión del ejercicio de la actividad, mas si se tiene en cuenta la apertura a la sexualidad que se ha vivido en las últimas décadas como respuesta una exteriorización de la intimidad en el escenario de lo público (Arendt, 2005). 
Edlund et al. (2002) indican como factores que influencian el desarrollo de este mercado:

1) Los altos ingresos que genera esta actividad a quien la desarrolla, ya que, por ejemplo, para el caso de Indonesia los ingresos por el desarrollo del mercado del sexo superan los ingresos de otras actividades, inclusive algunas que requieren altos niveles de capacitación.

2) El excedente poblacional de hombres incrementa la tendencia al trabajo sexual, como lo han demostrado estudios en campamentos mineros en donde los hombres superan a las mujeres en forma masiva.

3) La excesiva demanda masculina empuja la oferta de mujeres hacia el mercado del sexo, argumento sustentado en que, para algunos hombres, es más rentable pagar por un servicio sexual que mantener un matrimonio, teniendo en cuenta el caso de un temporal excedente de hombres en un lugar, ya que su paso transitorio les impide proyectar relaciones estables y duraderas, siendo el caso por ejemplo del turismo sexual.

4) El trabajo sexual ha sido vinculado a condiciones de pobreza por ingresos, lo que ha implicado para las mujeres que carecen de una entrada al mercado laboral por deficiencias, por ejemplo en la calidad educativa, de ejercer este trabajo como una salida a las necesidades económicas, lo cual implica que existe mayor mercado de sexo en países en vía de desarrollo.

Cabe mencionar, como ya lo han enunciado autores como Edlund et al. (2002), que existe una tendencia de desequilibrio demográfico frente al género que explica el desarrollo de este mercado, lo cual, en términos de Rubio (2006), explica la aceptación social del fenómeno y la legislación que sobre ella se deriva. 
Según Rubio (2006), las oleadas de trabajo sexual han estado relacionadas, más que con la situación económica, política o social, con los desequilibrios demográficos, y más específicamente a movimientos migratorios con los que se rompe el equilibrio de géneros en la población. Desde esta perspectiva se plantean dos escenarios demográficos fundamentales que explican esta tendencia.

Un primer escenario que presenta un exceso de hombres ocasionado por el desplazamiento de hombres solteros que buscan fortuna en alguna región determinada, siendo necesario tan solo el restablecimiento del equilibrio demográfico para la reducción de la prestación de servicios sexuales remunerados. El exceso de hombres de forma transitoria, también se ve por cuenta de cuarteles, campamentos o contingentes militares, "un ejemplo de este escenario, fértil como pocos para la prostitución, lo constituyen las bases militares estadounidenses en el Pacífico o en España" (Rubio, 2006, p. 5).

El segundo escenario se da con un exceso de mujeres solteras que fluyen hacia los centros urbanos, como el caso colombiano a mediados del siglo $\mathrm{XX}$, el cual se podría denominar como trabajo sexual por inmigración femenina a la metrópoli. Bajo este escenario, el trabajo sexual se ha confundido bajo un esquema patriarcal, con oficios tradicionalmente considerados como femeninos. Entonces, en un escenario contrario al primero en donde no hay escasez de mujeres, el ejercicio de la actividad presenta menor uso de la violencia, pero se presenta un mayor rechazo y estigmatización social al fenómeno; en gran parte, porque se enfrenta a la institución del matrimonio o de la pareja estable.

De acuerdo a lo anterior, Edlund en su artículo llamado "Sex and the City" (2005), demuestra para el caso de Suecia entre mayores ingresos por parte de los hombres en zonas urbanas, las mujeres jóvenes superará a los hombres jóvenes; esto explicado porque en las zonas urbanas se ofrecen mejores ingresos para 
trabajos calificados suponiendo que hay más hombres calificados que mujeres, resultando ser un incentivo para el aumento o excedente de mujeres o de una combinación del mejoramiento del mercado laboral y de las posibilidades de contraer matrimonio en un escenario en el que los hombres valoran las características asociadas a la fecundidad femenina, como la juventud, mientras que las mujeres buscan seguridad financiera cuando eligen a una pareja. De tal forma que si esta clase de hombres se encuentra en las zonas urbanas, podría explicar el exceso de mujeres en la ciudad.

\section{Economía y Derecho en el mercado del sexo}

Las discusiones que se han dado desde la economía, deben desprenderse del plano moralista que como lo señala Gonzales (2000) lo único que logra es imponer una coraza que impide analizar las motivaciones subyacentes a la elección y a la acción correspondiente; así mismo como lo señaló Posner (1992) en su investigación sobre la maximización de la Riqueza, no es necesario argumentar posiciones morales, en este caso la prostitución ya que sería poco realista suponer que se puede convencer a alguien de argumentos contrarios a su visión cuando esta nunca necesitó de argumentos para ser convencida de ella. En otros términos, lo que propone Posner, es que no se puede convencer mediante argumentos a alguien de dejar sus construcciones morales, más que por la experiencia.

Para el mercado sexual, la economía se ha alejado de estos supuestos morales en aras de verificar tendencias y supuestos que intentan explicar el desarrollo del fenómeno más allá de prejuicios moralistas. En estos términos, hablar de un mercado ilegal del sexo por sus implicaciones morales podría traer inconvenientes en términos de eficiencia como lo ha expuesto Gonzales (2000) al describir los meta-ordenamientos. 
Desde la perspectiva de los institucionalistas, si existe legalidad se constituye en el meta-ordenamiento de los agentes que participan en el mercado logrando transparencia y garantizando que los mercados operen eficientemente, por lo que un mercado ilegal como lo podrían ser los modelos abolicionistas frente al trabajo sexual, representaría en términos de mercado una pérdida de eficiencia; un modelo reglamentarista o inclusive legalista del mercado del sexo operaría con fluidez si los contratos con los agentes son aceptados, adquiriendo un estatuto privilegiado por cuenta de las instituciones que regulan las interacciones sociales dejando de lado problemas como la incertidumbre e inclusive determinando las decisiones de los agentes que se encuentran en el mercado (Gonzales, 2000).

Es importante señalar que frente al comercio sexual, por sus implicaciones en términos de la moralidad y del ámbito legal como se percibe en algunos modelos normativos, los agentes prefieren actuar bajo el marco de la legalidad como se ha expuesto, por cuenta de una mayor eficiencia de mercado. Según Gonzales (2000, p.41) "Las personas no solo explicitan sus preferencias sino que también definen su posición frente a la norma". Para mostrar gráficamente las repercusiones de una práctica ilegal y el costo de removerla, Gonzales (2000) expone la siguiente grafica:

\section{GRAFICO 3.}

\section{COMPORTAMIENTO DEL COSTO SOCIAL MARGINAL}

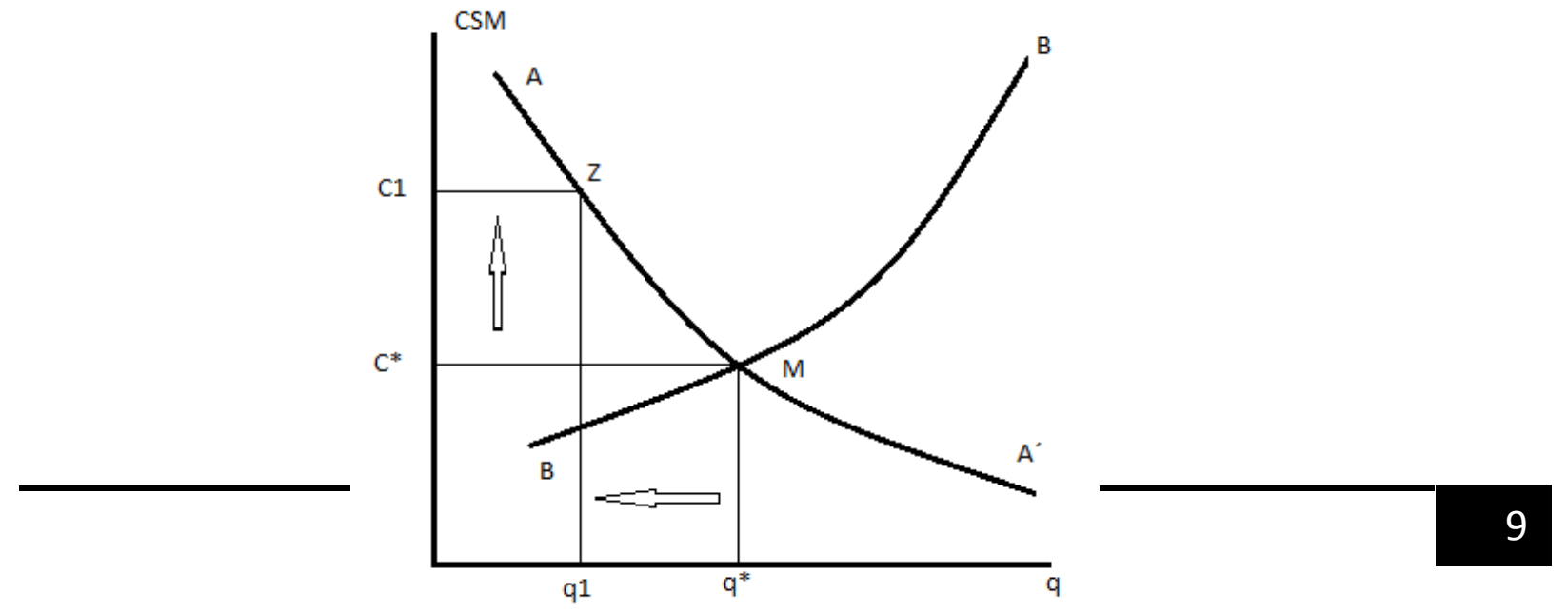


Fuente: Gonzáles, J. I. (2000). La ilegalidad, los meta-ordenamientos y las meta-preferencias. Revista de economía institucional.

En el eje horizontal se muestra el monto de la actividad ilegal (q); en el eje vertical se muestra el costo social marginal (CSM). En este plano la curva $A A^{\prime}$ representa el costo de remover una práctica ilegal, en este caso un modelo prohibicionista, abolicionista del comercio sexual y la curva BB' corresponde al costo de lo ilegal; de tal forma, se puede plantear que para la ilegalidad aumentarán los ingresos en la medida en que aumente el costo social marginal, mientras que la disminución de la ilegalidad de $\mathrm{q}^{\star}$ a $\mathrm{q} 1$ implica un aumento de los costos de $\mathrm{C}^{\star}$ a $\mathrm{C} 1$.

Esta grafica también permite comprender la razón por la cual el nivel óptimo no debe ser igual a cero (0) en el caso de la actividad ilegal; así mismo, como no es conveniente que sea totalmente legal por los costos sociales que implicaría no solo en términos monetarios, para el caso del comercio sexual, un mercado institucionalizado, vigilado o regulado para disminuir posibles costos sociales, debido a que el desarrollo del caso del trabajo sexual a una legalidad de toda la actividad, podría implicar por ejemplo un crecimiento descontrolado de fenómenos ilegales como la explotación sexual de niños, niñas y adolescentes aumentando los costos sociales. "Como la ilegalidad no es cero, la sociedad debe soportarla a un costo $C^{*}$. El punto $M$ es de equilibrio porque el costo de remover lo ilegal es igual a su costo social. El punto M significa que la sociedad termina conviviendo con algún grado de ilegalidad." (Gonzales, 2000, p. 48).

Ahora bien, considerando lo mencionado acerca del mercado del sexo y su relación con los mercados en el contexto legal, es necesario establecer la relación 
en el marco teórico de la economía y su correspondencia entre el trabajo del sexo, factores explicativos del funcionamiento del mercado sexual.

\section{El mercado del sexo desde la perspectiva económica}

El mercado del sexo representa toda una variedad de dinámicas que se insertan dentro del campo del análisis económico, Cuevas (2000) menciona que este mercado optimiza en términos económicos la prestación de un servicio sexual debido a los bajos costos de transacción, eliminando lo que implicaría un trueque en una relación afectiva subiendo los costos asociados ya sea en términos de tiempo, disposición o dinero.

Para Posner (1992) y Edlund et al. (2002), los hombres y la relación de tipo sexual con las mujeres representa un costo, mientras que para las mujeres representa un precio, basándose en una mayor demanda masculina y promiscuidad como lo evidencian las prácticas sexuales en la población de los EE.UU. en donde el 18 por ciento de los varones ha pagado alguna vez por tener sexo con una mujer, mientras que tan solo el 2 por ciento de las mujeres ha pagado por un hombre (Edlund et al., 2002). De esto, surge un costo neto positivo para los hombres y un ingreso neto positivo para las mujeres, generando un mercado en el que se deben encontrar la oferta y la demanda. Partiendo de esta base, hay un punto de análisis el cual ha sido de especial interés para los economistas y es el alto precio de un servicio sexual.

Se ha cuestionado la importante suma de dinero que puede percibir un/a trabajador/a del sexo, al respecto Edlund et al. (2002) indican que en Suecia, en 1998, quien se dedicaba al trabajo sexual percibía cerca de 1.750 dólares por día, valor semejante a los ingresos de un mes en un puesto de trabajo no calificado. En Letonia, según fuentes de los mismos autores, quien ejercía la actividad 
percibía un promedio de 5.000 dólares por mes, lo cual representaría 20 veces el salario medio de este país.

Para Levitt y Ventakesh (2007) el alto precio está determinado por los riesgos a los que se está expuesto, factor que representa la principal explicación del alto costo del servicio sexual dado en algunos casos también por el pago asociado al proxeneta el cual parece generar, según los autores, un efecto positivo para quien ofrece el servicio por cuenta de la protección que brinda; además porque, según datos de su investigación, quienes trabajan con proxenetas perciben mayores ingresos.

En este punto es conveniente mencionar que en el mercado del sexo existe una segmentación amplia que complejiza su análisis, partiendo desde las personas que ofrecen sexo en la calle, las que lo hacen en un establecimiento moralmente no aceptado, en hoteles, hasta las que lo hacen desde su presentación en páginas de internet o prepagos, entre otras tipologías; sin embargo, la estructura del mercado del sexo femenino ha sido similar a través del tiempo y en regiones geográficas, a partir del segmento más precario en el que se encuentran mujeres que ofrecen sus servicios en los espacios mencionados. Diferenciación que incluye en su precio la mejor apariencia, juventud, grado de anonimato, nivel educativo y reputación de ambas partes. (Edlund et al., 2002).

El mercado del sexo desde el institucionalismo.

tAlgunas visiones contemporáneas han expuesto el mercado del sexo desde el Algunas visiones contemporáneas han expuesto el mercado del sexo desde el institucionalismo y sus repercusiones en el plano de la legalidad representado por uno de sus fundadores, Douglas $C$. North, quien junto con Roger Leroy en su obra titulada "El análisis económico de la usura, el crimen, la pobreza" (1971), expusieron algunas de las características del mercado del sexo para comprender su funcionamiento desde las mismas leyes del mercado que ya promulgaba con 
antelación Adam Smith a diferencia que se incorporó su perspectiva teórica basada en la fuerza de las instituciones sobre el mercado.

Dichos autores exponen que el trabajo sexual tiene como los demás servicios dos dimensiones: calidad y cantidad, las cuales pueden variar una en función de la otra; es decir, en aumento de la calidad disminuye la cantidad y viceversa. En este sentido, North y Leroy (1971) al referirse al mercado del sexo exponen:

"La calidad del servicio es, entre otras cosas, una función de a) la experiencia (inversión en capital humano); b) las características innatas del proveedor de los servicios, como lo son la apariencia y la inteligencia y c) los gastos corrientes del trabajo, como la cantidad gastada en la apariencia, la salud y el medio ambiente." (North, et al., 1971, p.42).

En las características mencionadas se puede, según sugerencias de los autores, realizar una sustitución de algunas como, por ejemplo, en el caso de que una mujer nazca con características innatas que atraigan a su clientela, puede sustituir la experiencia.

Los autores consideran que el mercado del sexo en el marco de la legalidad permite que la información sea de fácil acceso asemejándose a un modelo de competencia perfecta en donde la información llega a ser simétrica, de tal forma que si en algún establecimiento se conocía de la presencia de algún riesgo de contraer enfermedades venéreas, sus precios caerían drásticamente por la reticencia de los clientes a frecuentar un sitio en donde se especulan estos riesgos.

Siendo este el panorama en un contexto de legalidad, los oferentes no incluyen dentro de los costos asociados a la prestación del servicio un factor "riesgo", debido a que la minimización de efectos negativos a la clientela como de costos 
asociados al ocultamiento de la actividad desembocaba en un precio que no incluye costos a la función de precio del servicio sexual.

Según North et al. (1971), el costo del negocio ha aumentado por cuenta de la inclusión de nuevos costos asociados al factor "riesgo", como las implicaciones de trabajar en la calle, lo que supone el peligro de ser arrestadas por la policía y en caso de ser arrestadas pagar sobornos. En un escenario de ilegalidad, las economías de escala que se favorecían con bajos costos de "accesorios" como el ambiente atractivo y cómodo a los consumidores, ya no se favorecían percibiendo un menor ingreso por lo cual a un costo de prestación de servicios sexuales en un nuevo contexto de ilegalidad las prestadoras de servicios ya no estarían dispuestas a ofrecer lo mismo por el precio anterior debido al incremento de los costos asociados.

Con respecto al comportamiento de la demanda en un contexto de ilegalidad, señalan North et al. (1971), varias consecuencias, como la pérdida de confianza por parte de los demandantes frente a la adquisición de una enfermedad venérea, por poseer escasa información y a su vez porque las oferentes particulares podrían bajar su calidad, en este caso estaría asociado a una posible enfermedad y en el caso de los sitios en los que se conociera sobre la existencia de algún riesgo perderían considerablemente demanda.

En este escenario de ilegalidad, la información sufre un cambio drástico hacia la asimetría lo que implica una subida de precios de la oferta, segmentando el mercado con discriminación de precios hacia los que pueden pagar por el servicio y por la seguridad de la calidad de la oferente y, por otra parte, los que pagan un servicio asumiendo el riesgo de la pérdida de calidad o en otros términos asumen los demandantes el riesgo de contraer una enfermedad venera por carencia en la información. Dicho riesgo que pueden asumir algunos demandantes tiene otras 
posibles implicaciones en términos de costos sociales, pues la enfermedad puede transmitirse en otros entornos fuera del mercado sexual.

De las nuevas fronteras de la economía a la prostitución

Desde una visión de libre mercado McKenzie y Tullock (1980) han hecho uso de las herramientas de la teoría económica para explicar el funcionamiento del mercado del sexo, ya que como mencionan, el estudio económico generalmente se ha ubicado en espacios netamente mercantiles y el imaginario sobre la ciencia económica ha desplazado su mirada sobre asuntos de esta índole, pero la incursión de la teoría económica en otros campos ha abierto nuevos escenarios de aprendizaje y conocimiento sobre el comportamiento humano, partiendo del principio que los seres humanos actúan bajo un objetivo, es decir, sus actuaciones están dirigidas por motivaciones abriendo el camino hacia la exploración de caminos inesperados de la conducta económica de los individuos.

Consecuentemente con los principios que plantea la economía se puede asociar a teorías aplicables al campo de la sexualidad, especialmente al campo del mercado del sexo.

En este orden, se aplica el campo de la teoría económica debido a que la utilidad que representa la prestación de un servicio sexual, ya sea así mismo o a otras, está determinado en un consumidor racional por una función de demanda que está en función inversa del precio, lo cual gráficamente se entiende como la tradicional curva de demanda que tiene una pendiente negativa. Este comportamiento supone que el consumidor estaría dispuesto a consumir un servicio sexual en cierta cantidad relacionado con la variación del precio que supone el servicio. Según McKenzie et al. (1980) la causa de esta interrelación (Cantidad /precio) esta explicada gracias a que el individuo racional consumirá 
sexo hasta el punto en que su beneficio marginal se iguale a los costos marginales.

Es decir,

$$
\begin{gathered}
\text { UMs } / \mathrm{Ps}=\mathrm{UMa} / \mathrm{Pa}=\mathrm{UMn} / \mathrm{Pn} \text {, en donde: } \\
\qquad \begin{array}{c}
\mathrm{UM}=\text { Utilidad marginal } \\
\mathrm{P}=\text { precio } \\
\mathrm{s}=\text { Sexo }
\end{array} \\
\text { a y } \mathrm{n}=\text { otras mercancías }
\end{gathered}
$$

De acuerdo con estos principios básicos de la economía, se puede establecer con claridad como el mercado del sexo juega dentro de unas reglas como cualquier otro bien o servicio de la economía:

Con un aumento de precio, el/la trabajador/a del sexo, no solamente puede ofrecer menos servicios sino que puede que la cantidad que se le demande caiga hasta el extremo de que haga disminuir sus ingresos totales, si tiene una cierta cantidad de competidores/ras disponibles. Los ingresos pueden aumentar si la demanda a la que se enfrenta es inelástica. (McKenzie et al., 1980).

Según otras investigaciones de McKenzie et al. (1980), se han revelado que los grupos de rentas más altas consumen más sexo que los de rentas inferiores, explicado por cuenta del posible diferencial en educación que les permite a quienes tienen rentas más elevadas tener una mentalidad más abierta respecto del sexo lo cual les produce menores inconvenientes o problemas morales 0 psicológicos frente a su sexualidad.

Entender el sexo como un intercambio mas allá de las funciones básicas de reproducción, implica entender que en cualquier tipo de relación sexual existirán intercambios implícitos que en algunas ocasiones tienen un precio, en términos 
monetarios, como en el caso del mercado del sexo, pero en otro tipo de relaciones como las matrimoniales los intercambios sexuales cambian de forma de pago, llevando el precio a otras dimensiones no monetarias como las afectivas; es decir, si el trabajo sexual se paga a un precio monetario por la adquisición del servicio, en una relación matrimonial se paga con fidelidad, compromiso, lealtad y otra cantidad de características que son pactadas como precio para la prestación del servicio sexual en el momento en el que el intercambio sobrepasa el deseo sexual de cualquiera de las personas involucradas en el pacto.

Para entender el mercado del sexo, McKenzie et al. (1980) asume los principios básicos de las reglas de un mercado en condiciones de competencia perfecta, lo que implica que la cantidad ofrecida de sexo aumentará en la medida en que aumente su precio, a su vez que una disminución en el precio, producirá un aumento de la cantidad demandada se sexo.

En el contexto de la sexualidad, cabe mencionar que tras los cambios que enunció la liberación femenina desde los años sesenta, los costos asociados al sexo han disminuido para las mujeres por cuenta de la generalización de los anticonceptivos, de los estigmas morales, de las crisis del matrimonio, de la cada vez más aceptación de los abortos, entre otros tantos costos que han generado un crecimiento de la oferta de sexo por parte de las mujeres, lo que conduce a una reducción del precio de las unidades de sexo ofrecidas, aumentando de esta forma la cantidad de sexo general.

De esta forma, el mercado del sexo toma sentido en la medida que "el precio de la prostituta, aunque llegue inclusive a las siete mil pesetas, puede ser mucho más bajo que el coste que tendría que soportar un hombre (o una mujer) para obtener el mismo placer de fuentes mas "legitimas". Uno puede pagar las siete mil pesetas, $y$ al hacerlo se ahorra el tiempo que hace falta para seducir a la/el profesional." (McKenzie et al., 1980, p.99). Lo anterior, implicaría flores, 
invitaciones, regalos y demás costos asociados al galanteo necesario que conduzcan a la relación sexual, sin contar con el tiempo necesario para que esto ocurra.

Esto abre el campo del trabajo sexual como una manifestación de la racionalidad de las necesidades sexuales en donde el precio, esta vez monetario, se manifiesta en una oferta que resulta ser más conveniente para quien en otro mercado como el noviazgo o el matrimonio perciben un mayor precio.

\section{Mercado laboral y género}

Se podrá encontrar a lo largo de los estudios del mercado del sexo una gran cantidad de investigaciones sobre el trabajo sexual femenino, como si esta fuera la única categorización existente en este mercado, hecho que no es gratuito, no por tener el sexo femenino una inclinación natural hacia este fenómeno, ni así mismo los hombres una inclinación natural a la demanda de servicios sexuales remunerados, como se podrá evidenciar porque la estructura social se ha basado en una división sexual del trabajo que ha conducido a que la mujer quede replegada respecto del hombre en el mercado laboral.

En el Tratado sobre la Familia (1991), Becker ha señalado de acuerdo con las ventajas comparativas que un hogar eficiente se da cuando las partes se especializan invirtiendo cada miembro su tiempo y capital humano, equilibrándose una distribución entre el tiempo y los beneficios, explicado implícitamente por la necesidad de la mujer de quedarse en casa mientras que el hombre forma parte de la fuerza productiva, logrando de esta forma maximizar los beneficios al interior de la familia; por otra parte, es la mujer, de acuerdo con la exposición de Becker (1991) quien debe dedicarse a la labores domésticas ya que los salarios de las mujeres son inferiores a los del hombre y porque las mujeres invierten menores cantidades de capital humano de mercado. 
Las diferencias de salario según lo señala Cohen (1985) son bien conocidas, a pesar de que la economía no ha estado especialmente interesada en estudiar este fenómeno, aunque los neoclásicos hayan señalado en la misma ruta como Becker que las diferencias salariales están explicadas por la productividad, pero como lo señala Cohen (1985) la teoría neoclásica se basó en una economía perfectamente competitiva, siendo la discriminación un comportamiento irracional.

Así mismo, desde la aparición de la teoría del capital humano las explicaciones parecen seguir en la misma vía aplicando la teoría neoclásica, pero esta vez a fenómenos que no habían sido explicados en épocas precedentes. De acuerdo con lo anterior, Cohen (1985) señala que la teoría del capital humano no considera desleal esta diferencia salarial ya que las personas deciden libremente el nivel de inversión de capital que hacen en sí mismas, evidenciado por las decisiones que toman las mujeres en invertir menos años de trabajo que los hombres, lo cual da a los hombres mayores habilidades y por consiguiente una mayor productividad.

Para Anzorena (2009) los principios expuestos por Becker (1991) en los que describe las desigualdades de mercado entre hombres y mujeres no pueden considerarse como un principio que abarque todo el espectro de la estructura social ya que en la práctica "este enfoque sólo "racionaliza" o justifica el status quo, pero no lo explica" (Anzorena, 2009, p.6).

En esta misma vía Jelin (1978) ha señalado la discriminación de la cual es victima la mujer, especialmente en las áreas urbanas en donde se han definido algunos trabajos como adecuados para la mujer, mientras otros fuera de su alcance, quedando relegada a trabajos que extienden el rol domestico tradicional. De acuerdo con estas convenciones, se han definido tres diferentes ofertas de empleo femenino: 1) las que no poseen responsabilidades domesticas ya que no tienen o no hacen parte de un núcleo familiar o matrimonio; 2) las mujeres que solo se 
dedican a las labores domesticas, y 3) las mujeres que se dedican a las labores domesticas, además de estar insertas en el mercado laboral (Jelin, 1978).

\section{Conclusiones}

Desde el contexto económico, las explicaciones sobre los determinantes del mercado del sexo han estado caracterizadas por influencias externas como la pobreza por ingresos, la creciente movilización internacional, además de los importantes ingresos recibidos por quien ejerce la actividad en un marco en donde predomina la demanda masculina y la oferta femenina. Teniendo en cuenta las explicaciones que fundamentan la existencia del fenómeno se han señalado dos posiciones desde las cuales han estado presentes los estudios, oscilando entre el rechazo presente en la teoría feminista critica y su aceptación por parte de tendencias liberalizadoras que perciben esta actividad como un trabajo, que han dado origen a los enfoques jurídicos que se enmarcan en el prohibicionismo, abolicionismo, reglamentarismo y legalización del fenómeno.

Por otra parte, este escenario plantea la necesidad académica de profundizar en estudios relacionados futuros no solo con el comercio sexual y sus implicaciones, sino con el nuevo escenario de incorporación de la mujer en el mercado laboral, trastornando las visiones tradicionales de la economía, por lo menos a lo referente al papel de la familia y a la división social del trabajo, permitiendo un entendimiento de una realidad social que permita atinar de forma adecuada el fenómeno del mercado sexual desembocando en el mejoramiento de las condiciones de calidad de vida, además de condiciones laborales y sociales, asegurando un futuro en el que se garanticen los derechos en un marco democrático que incluya la diferencia como aspecto fundamental de las sociedades modernas, lo cual se puede lograr con una reformulación y revisión de los tradicionales paradigmas positivistas que han dejado un plano epistemológico 
excluyente con los nuevos discursos que permiten la incorporación de visiones de género, amplios, flexibles y no excluyentes.

Por último, es necesario señalar que comprender el mercado del sexo desde una perspectiva económica se ajusta a una necesidad estructural de la sociedad, no solo en términos de criterios como los de eficiencia o eficacia, sino en el campo social desde una visión diferencial que permita la estructuración de fundamentos que sirvan de insumo para las políticas públicas que den cuenta de las necesidades de la población vinculada dejando visiones asistencialistas y victimarias que desconocen el libre ejercicio de la actividad, haciendo uso del bagaje teórico de la economía sumándose al desarrollo de las ciencias sociales frente al tema.

\section{Referencias bibliográficas}

Anzorena, C. (2009). El ¿retorno? del Tratado sobre la Familia de Gary BeckerAlgunas reflexiones en torno a los criterios de eficiencia que legitiman los planes compensatorios de fines de la década de 1990. Kairos, (24), 1-0. Tomado de http://dialnet.unirioja.es/servlet/articulo?codigo=3101150

Arendt, Hannah. (2005). La condición Humana. Barcelona: Paidos.

Becker, G.S. (1993). Human Capital: A theoretical and empirical analysis, with special reference to education (p. 416). University of Chicago Press. Tomado de http://ecsocman.hse.ru/text/19164330/

Becker, G.S., Murphy, K. M., \& Grossman, M. (2008). El mercado de bienes ilegales: el caso de la droga. Revista de economía institucional, 8(15), 17-42. Tomado de http://foros.uexternado.edu.co/ecoinstitucional/index.php/ecoins/article/view/44 
Becker, Gary S, Landes, E. M., \& Michael, R. T. (1977). An Economic Analysis of Marital Instability. Journal of Political Economy, 85(6), 1141-1187.

Cohen, M. (1985). The Razor's Edge Invisible: Feminism's Effect on Economics. International Journal of Women's Studies, 8(2), 286-98. Tomado de http://www.sfu.ca/ mcohen/publications/women/Razor's Edge.pdf

Contraloría de Bogotá D.C. (2004). Estudio sectorial "la prostitución como problemática social en el distrito capital." Historia. Bogotá.

Cuevas, H. (2000). La teoría económica, el afecto y la familia, 2, 13-36. Tomado de http://papers.ssrn.com/sol3/papers.cfm?abstract id=446460

Edlund, L. (2005). Sex and the City. Scandinavian Journal of Economics, 107(1), 25-44. doi:10.1111/j.1467-9442.2005.00393.x

Edlund, L., \& Korn, E. (2002). A Theory of Prostitution. Journal of Political Economy, 110(1), 181-214.

Gonzáles, J. I. (2000). La ilegalidad, los meta-ordenamientos y las metapreferencias. Revista de economía institucional, 2. Tomado de http://redalyc.uaemex.mx/redalyc/html/419/41900203/41900203 1.html

Jelin, E. (1978). La Mujer y el Mercado de Trabajo Urbano (2nd ed.). Estudios Cedes.

Levitt, S. D., \& Venkatesh, S. A. (2007). An empirical analysis of street-level prostitution. Preliminary draft, September, (September). Tomado de http://economics.uchicago.edu/pdf/Prostitution 5.pdf?q=venkatesh 
McKenzie, Richard y Tullock, Gordon (1980). La nueva frontera de la economía. Centro de Estudios y Comunicación Económica: Madrid

Mill, J. S. (1869). La esclavitud femenina. Biblioteca de la mujer, 2. Tomado de http://books.google.com.co/books/about/La_esclavitud_femenina.html?id=FWEiAQ AAIAAJ\&redir_esc $=y$

North, Douglass Leroy M, Roger. (1971) En análisis económico de la usura, el crimen, la pobreza, etcétera. Fondo de Cultura Económica: México D.F

Posner, R. A. (1992). Sex and Reason. Cambridge (MA): Harvard University

Rubio, M. (2006). La prostitución latinoamericana y su incidencia en España. Instituto universitario de investigación sobre seguridad interior, 1-36. Tomado de www.uned.es/investigacion/publicaciones/Cuadernillo_abril2006.pdf

Rubio, M. (2008). La pandilla proxeneta: violencia y prostitución juvenil en Centroamérica. Revista Latinoamericana de Seguridad Ciudadana. Tomado de http://www.flacsoandes.org/urvio/img/Urv4_Inv4.pdf

Rubio, M. (2008). Ni puta ni trabajadora sexual: prostituta. Borradores de Método, (50). Tomado de http://scholar.google.com/scholar?hl=en\&btnG=Search\&q=intitle:Ni+puta+ni+trabaj adora+sexual+:+prostituta\#0

Salcedo, E., Rubio, M., \& Beltran, I. (2008). Entre sexo y dinero: Una revisión de las principales posturas e interpretaciones de la prostitución. Borradores de Método. Tomado de http://ideas.repec.org/p/col/000133/005464.html 
Smith, A. (2010). Investigación de la naturaleza y causas de la riqueza de las naciones. (1794 Oficina de la Viuda e Hijos de Santander, Ed.). Madrid: Universidad Complutense de Madrid.

Tirado Misael (2011). Implicaciones del Trabajo Sexual como una actividad legalmente reconocida: Un acercamiento al trabajo sexual desde sus implicaciones sociológicas y económicas. Revista Prolegómenos: Derechos y Valores. Vol. XIV-27. Bogotá: Universidad Militar Nueva Granada

Tirado, Misael (2010). Comercio Sexual: Una mirada desde la sociología jurídica. Instituto Internacional de Derecho y Sociedad - Lima Perú, Fundación FEFSA Bogotá Colombia. 